J Ästhet Chir 2009 - 2:6-7

DOI 10.1007/s12631-009-0021-3

Online publiziert: 28. Januar 2009

๑) Springer Medizin Verlag 2009

H.G. Bull

St. Josefshospital Uerdingen, Krefeld

\title{
Jetzt jede Ausgabe mit Schwerpunktthema
}

Sehr geehrte Frau Kollegin, sehr geehrter Herr Kollege,

das „Journal für Ästhetische Chirurgie“, dessen erste Ausgabe 2009 Ihnen jetzt vorliegt, wird sich zukünftig in jeder Ausgabe einem Themenschwerpunkt widmen (• Tab. 1).

Erster Themenschwerpunkt ist die Volumensubstitution von Weichgeweben, die sogenannte Fillertherapie. Durch die Originalarbeiten namhafter Autoren wird ein wissenschaftlich fundierter Überblick über den aktuellen Stand von Klinik und Forschung vermittelt. Dabei wird insbesondere klar und eindeutig herausgearbeitet, dass es sich bei der „Fillerbehandlung" um einen ernsthaften dermatochirurgischen Eingriff handelt, der nur und ausschließlich durch erfahrene und in dieser speziellen Therapie ausgebildete Fachärzte erfolgen sollte. Dies muss erwähnt werden, weil in der Öffentlichkeit - und insbesondere in der Regenbogenpresse ein völlig anderes Bild gezeichnet wird. Leider muss in diesem Zusammenhang auch festgestellt werden, dass es sich bei den Präparaten nicht um Medikamente sondern um sogenannte CE-zertifizierte Medizinprodukte handelt, die häufig keine oder nur eine unzureichende wissenschaftliche Grundlagenforschung vor der „Markteinführung“ vorzuweisen haben. Aus diesem Grund fordert die GÄCD schon seit langer Zeit die Einordnung der Fillersubstanzen als Medikamente.

Themenschwerpunkt der zweiten Ausgabe 2009 wird die Chirurgie des al- ternden Gesichts, vom Stirnlift über das Facelift bis zum Halslifting, sein.

Für die dritte Ausgabe steht die Nasenchirurgie in ihrer funktionellen und ästhetischen Bedeutung im Mittelpunkt.

Die vierte Ausgabe wird sich dann thematisch der Lidchirurgie in Funktion und Ästhetik widmen.

So hoffen wir, dass wir mit diesen vier Themenschwerpunkten für das Jahr 2009 interessante und für die ästhetische Chirurgie insgesamt wichtige Gebiete abhandeln können.

Ich wünsche den Mitgliedern der Gesellschaft und allen Leserinnen und Lesern viel Spaß beim Lesen und ein glückliches und erfolgreiches Jahr 2009.

Ihr

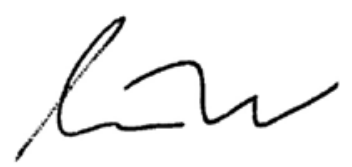

Prof. Dr. Dr. med. H.G. Bull Präsident der GÄCD

\section{Korrespondenzadresse}

Prof. Dr. Dr. H.G. Bull

St. Josefshospital Uerdingen

Kurfürstenstraße 69 ,

47829 Krefeld

bull.mkg@stjosef.de

Geschäftsstelle der GÄCD

Königsteiner Str. 55a

65812 Bad Soden a.T. 
Hier steht eine Anzeige.

算 Springer 